\title{
Impending Paradoxical Embolism in Pregnancy: Report of A Case
}

\author{
Tateo Saverio*1, Endrizzi Barbara², Graffigna Angelo ${ }^{2}$ and Mereu Liliana ${ }^{2}$ \\ ${ }^{1} 1$ Department of Obstetrics and Gynecology of Santa Chiara Hospital, Italy \\ ${ }^{2}$ Department of Cardiosurgery of Santa Chiara Hospital, Italy
}

*Corresponding author: Tateo Saverio, Department of Obstetrics and Gynecology of Santa Chiara Hospital, Trento, Italy

\section{ARTICLE INFO}

Received: 幽 October 09, 2019

Published: 幽October 21, 2019

Citation: Tateo Saverio, Endrizzi Barbara, Graffigna Angelo, Mereu Liliana. Impending Paradoxical Embolism in Pregnancy: Report of A Case. Biomed J Sci \& Tech Res 22(2)-2019. BJSTR. MS.ID.003718.

Keywords: Thromboembolectomy; Systemic heparinization; Eterozygosis

ABSTRACT

Atrial thrombosis is a rare complication of Venous Thrombo-Embolism (VTE) and is associated with a higher mortality rate. In presence of a patent foramen ovale, a thrombus can be trapped in (thrombus straddling in foramen ovale: TSFO), constituting a high-risk condition named Impending Paradoxical Embolism (IPDE). No previous case of IPDE in pregnancy has been reported. We describe a case of a 32-year-old woman, at 25 weeks of gestation following in vitro fertilization, with pulmonary embolism and TSFO. Open heart surgery, in order to remove a $25 \mathrm{~cm}$ thrombus and repair the septal defect, was successfully performed after failure of medical therapy. Cardiac surgery on cardiopulmonary bypass involved the use of high flow, high pressure, normo thermic bypass in combination with continuous fetal monitoring during the procedure. In case of impending paradoxical embolism (IPDE), the optimal treatment is controversial and in pregnancy fetal risk should be considered in relation to gestational age.
\end{abstract}

Abbreviations: DVT: Deep Venous Thrombosis; PE: Pulmonary Embolism; TSFO: Thrombus Straddling in Foramen Ovale; IPDE: Impending Paradoxical Embolism

\section{Introduction}

Thrombo-embolic disease is a major cause of maternal morbidity and mortality during pregnancy and puerperium. The age-adjusted risk of VTE is from four to five times higher in pregnant versus non-pregnant women [1,2]; the incidence ranges approximately from 0.76 to 1.72 per 1000 deliveries, and the mortality rate ranges from 1.1 to 1.5 per 100.000 deliveries [1,2].

The migration of a deep venous thrombosis (DVT) through the vena cava and right heart cavities is the leading cause of Pulmonary Embolism (PE). In the setting of massive PE, a foramen ovale can become patent and allow an intra cardiac thrombus to migrate toward the left atrium, producing paradoxical systemic embolization. If the thrombus is sufficiently large compared with the size of the foramen ovale, it can be trapped during its passage and straddle in the foramen (thrombus straddling in foramen ovale: TSFO), producing impending paradoxical embolism (IPDE). This is a high-risk situation, which carries a high mortality and requires emergency treatment [3]. We describe a case of IPDE occurred during the second trimester of pregnancy, requiring emergent surgical thromboembolectomy and foramen ovale closure on cardiopulmonary bypass after failure of medical therapy.

\section{Case Report}

A 32-year-old nulliparous woman presented at 25 weeks of gestation with intermittent thoracic pain and shortness of breath. The patient had complained early fatigability in the past 2 weeks, after a two hours plane travel. Pregnancy was obtained with in vitro fertilization and embryo transfer. Her past medical history was unremarkable. Her family history revealed a cousin affected by pulmonary embolism at young age. On examination, her blood pressure was $120 / 80$, pulse 110 beats/min, respiratory rate $16 / \mathrm{min}$, oxygen saturation $96 \%$ on room air. On cardiovascular examination, regular heart sounds with no murmurs were heard. An electrocardiogram showed sinus tachycardia and a T wave asymmetric inversion in V2, V3 e V4 pre cordial leads (acute right overload). 
A trans thoracic echocardiography was performed, and showed a sepiginous mobile right atrial mass, with an extremity adherent to the atrial septum, in the lower part (annulus ovalis); a specular mass was present in the left atrium, prolapsing through the mitral valve into the left ventricle, revealing a thrombus originating between the left and right atrium via PFO. The right ventricle appeared dilated (Tricuspid Annular Plane Systolic Excursion - TAPSE - $18 \mathrm{~mm}$ ), with tricuspid valve insufficiency $(2+/ 4+$ on Pulmonary Artery Pressure systolic - PAPs - 52 mmHg). Lower extremities Doppler ultrasound revealed a deep venous femoropopliteal thrombosis, in the presence of a collateral femoral superficial vein. Routine laboratory tests were normal, blood gas analysis on room air showed pH 7.49, pCO2 $26.5 \mathrm{mmHg}$ and p02 $74.9 \mathrm{mmHg}$ (respiratory alkalosis). Obstetric examination on admission confirmed a single normal ongoing pregnancy at 25 weeks of gestation; ultrasound showed normal fetal growth and no structural abnormalities; fetal heart monitoring was reassuring.

The patient was admitted in Cardiac Intensive Care Unit, and treatment was started with Heparin infusion. Two days later, a trans thoracic echocardiography showed enlargement of the thrombus, reaching the aortic valve. After a cardiac surgery, obstetric and anesthesiology consultation, a decision was made to proceed to surgery. The couple was counseled regarding the risk of fetal (16$33 \%$ ) and maternal mortality. Under systemic heparinization, cardiopulmonary bypass (CPB) was instituted through ascending aorta and bicaval cannulation. Cold cardioplegic arrest was established. The patient was maintained on CPB for $20 \mathrm{~min}$, the aortic cross clamp time was $12 \mathrm{~min}$. Systemic normo thermia was maintained. The right atrium was incised revealing a crossing atrial thrombus, $25 \mathrm{~cm}$ in length. The thrombus was removed and the patent foramen ovale was closed. During surgery, continuous fetal heart monitoring was performed, and it persisted reassuring.

Subsequent hospital course was unremarkable. She was discharged on enoxaparin $6000 \mathrm{UI}+5000 \mathrm{UI}$ and aspirin $50 \mathrm{mg}$ daily until the $36^{\text {th }}$ week of pregnancy. Subsequent obstetric course was uneventful. The patient delivered at 38 weeks by elective caesarean section a healthy girl infant (2590 gr, Apgar 9/9). Postoperatively warfarin was started and heparin was discontinued on achieving therapeutic levels of warfarin. Thrombophilic screening revealed eterozygosis for prothrombin gene G20210A mutation.

Seven years baby follow up proved normal development.

\section{Discussion}

In case of impending paradoxical embolism (IPDE), the optimal treatment is controversial. There is no evidence of superiority between different strategies such as thromboembolectomy, thrombolysis or anticoagulation; available literature is based mostly on a small number of case reports or observational studies. According to a recent review [3], surgical thromboembolectomy has lower mortality $(\mathrm{p}=0.04)$ and lower systemic embolism ( $p$ $=0.02$ ) than other treatment groups. On the other hand, cardiac surgery during pregnancy, especially if involving cardiopulmonary bypass, has been reported to carry significant maternal and fetal risk, of both preterm delivery and fetal mortality, and is tipically considered only after failure of medical therapy, or when medical therapy is not feasible [4-5].

To our knowledge, no previous case of IPDE in pregnancy has been described. The decision of proceeding to surgery has been made after failure of medical therapy, since it is known that cardiac surgery on cardiopulmonary bypass performed during pregnancy is associated with a fetal mortality rate ranging from 14.3 to $38.5 \%$ [5-7], while maternal mortality rate is reported to be similar to that of CPB in non-pregnant women (3\%), unless the surgery is performed on an emergent basis [8-10]. As far as fetal outcome is concerned, previous studies are not large enough to identify useful predictors [4]. According to some publications, fetal mortality appears to decline as cardiac surgery is delayed till the fetus is allowed to mature $[5,10]$; others claim that increased gestational age is linked to higher fetal morbidity [8].

Fetal losses also appear to be associated with urgent, highrisk cardiac surgery and maternal co-morbidities $[4,5,10]$, but cardiopulmonary bypass is probably the most remarkable determinant in pregnancy outcome, since it seems to be a strong stimulus to contraction genesis [11]. The use of hypothermia too, especially in the warming phase, seems to provoke contractile uterine activity, and seriously reduce placental functions [4,8, 11,12]. This is revealed by decelerations at continuous fetal heart rate monitoring during surgery, which should always be used when gestational age is greater than 24 weeks, according to several Authors $[5,11]$. Thus, if the fetus is mature enough and the planned maternal surgery is anticipated to be complicated or prolonged in length, delivery prior to CPB should be considered $[4,5,9,12]$.

If this is not feasible, strategies aimed at minimizing fetal risks should be used. These include maintaining uterine displacement to avoid aorto-caval compression starting from 20 weeks of gestation , minimizing intra operative blood loss, minimizing $\mathrm{CPB}$ times, using normo thermic $\mathrm{CPB}$, monitoring serum potassium concentration (goal $<5 \mathrm{mmol} / \mathrm{L}$ ), maintaining a high flow rate $\left(>2.4 \mathrm{~L} / \mathrm{min} / \mathrm{m}^{2}\right)$, and mean arterial pressures greater than 70 to $75 \mathrm{mmHg}$ [5]. Monitoring fetal heart rate with cardiotocography after 24 weeks of gestation [5] during CPB, allows to adjust the flow rate, the mean arterial pressure, and the maternal temperature in order to maintain fetal heart rates between 110 and 160 beats per minute $[5,13]$. An alternative way for fetal monitoring has been described, by using umbilical artery flow velocity by trans vaginal ultrasonography during the procedure [13].

\section{Conclusion}

Our work shows that cardiac surgery on cardiopulmonary bypass in pregnancy may be a safe also in extremely low gestational age. When surgery is necessary, the pregnant patient should be managed in a tertiary care centre by a multidisciplinary team, 
including cardiologists, cardiac surgeons, maternal fetal medicine specialists, anesthesiologists, and neonatologists in order to ensure the best outcome for both mother and fetus.

Strategies to minimize fetal risk in case of cardiac surgery with CPB are still not fully defined; actual clinical attitude involves: minimizing intra operative blood loss, maintaining uterine displacement to avoid aorto-caval compression after 20 weeks of gestation, using high flow normo thermic $\mathrm{CPB}$, minimizing $\mathrm{CPB}$ times and monitoring fetal heart rate with cardiotocography after 24 weeks of gestation.

\section{References}

1. James AH, Jamison MG, Brancazio LR, Myers ER (2006) Venous thromboembolism during pregnancy and the postpartum period incidence risk factors and mortality. Am J 194(5): 1311-1315.

2. Marik PE, Plante LA (2008) Venous thromboembolic disease and pregnancy. N Engl J Med 359(19): 2025-2033.

3. Myers PO, Bounameaux H, Panos A, Lerch R, Afksendiyos A (2010) Impending Paradoxical Embolism Systematic Review of Prognostic Factors and Treatment. CHEST 137(1): 164-170.

4. Barth WB (2009) Cardiac surgery in pregnancy. Clinical Obstetrics and Gynecology 52(4): 630-646.

5. John AS, Gurley F, Schaff HV, Warnes CA, Phillips SD, et al. (2011) Cardiopulmonary Bypass During Pregnancy. Ann Thorac Surg 91(4) 1191-1196.

\section{ISSN: 2574-1241}

DOI: 10.26717/BJSTR.2019.22.003718

Tateo Saverio. Biomed J Sci \& Tech Res

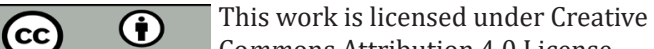

Submission Link: https://biomedres.us/submit-manuscript.php
6. Martin SR, Foley MR (2006) Intensive care in obstetrics: An evidencebased review. American Journal of Obstetrics and Gynecology 195(3): 673-689.

7. Sepehripour AH, Lo TT, Shipolini AR, McCormack DJ (2012) Can pregnant women be safely placed on cardiopulmonary bypass. Interactive CardioVascular and Thoracic Surgery 15(6): 1063-1070.

8. Patel A, Asopa S, Tang ATM, Ohri SK (2008) Cardiac Surgery during Pregnancy. Tex Heart Inst J 35(3): 307-312.

9. Parry AJ, Westaby S (1996) Cardiopulmonary Bypass During Pregnancy. Ann Thorac Surg 61: 1865-1869.

10. Weiss BM, Von Segesser LK, Alon E, Seifert B, Turina MI (1998) Outcome of cardiovascular surgery and pregnancy: A systematic review of the period 1984-1996. Am J Obstet Gynecol 179(6 pt 1): 1643-1653.

11. Pomini F, Mercogliano D, Cavalletti C, Caruso A, Pomini P (1996) Cardiopulmonary Bypass in Pregnancy. Ann Thorac Surg 61(1): 259268.

12. Yuan SM (2014) Indications for Cardiopulmonary Bypass During Pregnancy and Impact on Fetal Outcomes. Geburtsh Frauenheilk 74(1): $55-62$.

13. Mishra M, Sawhney R, Kumar A, Bapna KR, Kohli V, et al. (2014) Cardiac surgery during pregnancy: Continuous fetal monitoring using umbilical artery Doppler flow velocity indices. Annals of Cardiac Anaesthesia 17(1): 46-51.

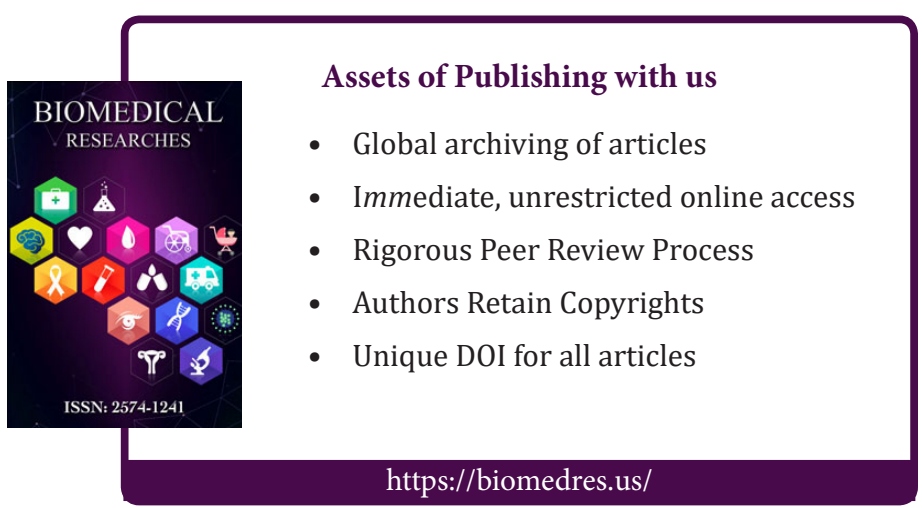

